\title{
Energetics of the AK13 semilocal Kohn-Sham exchange energy functional
}

\author{
A. Lindmaa* and R. Armiento ${ }^{\dagger}$ \\ Department of Physics, Chemistry and Biology (IFM), Linköping University, SE-581 83 Linköping, Sweden
}

(Received 7 April 2016; revised manuscript received 3 August 2016; published 27 October 2016)

\begin{abstract}
The recent nonempirical semilocal exchange functional of Armiento and Kümmel [Phys. Rev. Lett. 111, 036402 (2013)], AK13, incorporates a number of features reproduced by higher-order theory. The AK13 potential behaves analogously with the discontinuous jump associated with the derivative discontinuity at integer particle numbers. Recent works have established that AK13 gives a qualitatively improved orbital description compared to other semilocal methods, and reproduces a band structure closer to higher-order theory. However, its energies and energetics are inaccurate. The present work further investigates the deficiency in energetics. In addition to AK13 results, we find that applying the local-density approximation (LDA) non-self-consistently on the converged AK13 density gives very reasonable energetics with equilibrium lattice constants and bulk moduli well described across 13 systems. We also confirm that the attractive orbital features of AK13 are retained even after full structural relaxation. Hence, the deficient energetics cannot be a result of the AK13 orbitals having adversely affected the quality of the electron density compared to that of usual semilocal functionals; an improved orbital description and good energetics are not in opposition. This is also confirmed by direct calculation of the principal component of the electric field gradient. In addition, we prove that the non-self-consistent scheme is equivalent to using a single external-potential-dependent functional in an otherwise consistent, nonvariational Kohn-Sham density-functional theory (KS DFT) scheme. Furthermore, our results also demonstrate that, while an internally consistent KS functional is presently missing, non-self-consistent LDA on AK13 orbitals works as a practical nonempirical computational scheme to predict geometries, bulk moduli, while retaining the band structure features of AK13 at the computational cost of semi-local DFT.
\end{abstract}

DOI: 10.1103/PhysRevB.94.155143

\section{INTRODUCTION}

Density-functional theory (DFT) [1], in its standard formulation of Kohn and Sham (KS) [2], is a very successful approach to the many-electron problem. It is generally accurate, and requires relatively little computational effort, compared to, e.g., wave-function based methods. While it is exact in principle, its key ingredient, the exchange-correlation (xc) energy functional is in practical calculations approximated. Many viable approximations have been proposed.

A very common class of functionals rely solely on semilocal information in the density, i.e., $\nabla^{k} n$ to some order $k$, where $n(\mathbf{r})$ is the electronic density. Semilocal DFT gives good results for structural properties. It is well established that there are a number of highly relevant exchange features that semilocal DFT omits, such as the derivative discontinuity [3] and the relative offset between potentials for well-separated subsystems [4]. These are closely related to the self-interaction error and lead, e.g., to overdelocalized KS orbitals, and which arguably constitutes a major deficiency in semilocal approximations. The missing features are to a large degree restored in higher-order methods such as hybrids [5], exact exchange (EXX) within the optimized effective potential (OEP) method [6,7], and many-body perturbation theory (in particular, the GW approximation [8]). These methods, however, increase the computational cost. This is in many situations undesirable, and hence it is a worthwhile goal of broad interest for applications across a large number of

\footnotetext{
*alexander.lindmaa@liu.se

†rickard.armiento@liu.se
}

scientific fields to pursue a way to incorporate the exchange features into semilocal DFT methods.

Recently, one of us proposed an exchange-energy functional [9] (AK13) which, in several aspects mimics higherorder theory. Compared to the local-density approximation (LDA), and the widely used generalized-gradient approximation (GGA) functional of Perdew, Burke, and Ernzerhof [10] (PBE), it improves on the electronic structure and optical dielectric constants [11], which indicates that its KS orbitals resemble more the true quasiparticle electron states of the system. For atoms and small molecules, Cerceira et al. found an overall improvement of the first ionization potential as obtained from the highest occupied KS eigenvalue [12]. A recent all-electron study of solids [13] reveals that the orbitals obtained from the AK13 potential greatly improves on EXX total energies, as well as the general features of the potential shape. The combined picture from these works is that both the $\mathrm{KS}$ states and their respective eigenvalues (i.e., band structure) have generally improved. In the following, we refer to this as the improved orbital description of AK13. In spite of the fact that AK13 improves on the properties of orbitals, its energetics and total exchange energies are generally worse $[9,12]$. In particular, bulk Al gives an equilibrium lattice constant $6 \%$ larger than the exchange-only version of PBE. In the present work we further investigate this deficiency in energetics. We find that the average error is about $12 \%$ compared to the couple of percent error for typical semilocal functionals.

The AK13 functional differs significantly from usual functionals in generalized-gradient form (GGA). Its refinement factor is strongly divergent with the reduced density gradient. As a result it severely violates the local LiebOxford bound [14], and thus its deviation from the LDA exchange energy is unconstrained. Furthermore, far outside 
a finite system, the AK13 effective potential approaches by construction a finite nonzero asymptotic value that depends on the highest occupied Kohn-Sham eigenvalue. This feature affects the global energy scale [9]. With this background, one may ask if the deficiency in energetics stems from a misfeature directly in the AK13 electron density caused by a qualitative difference in the orbitals, or, if the deficiency instead primarily stems from the energies assigned by the AK13 functional to those densities. This is a relevant question, because the former option means the deficiency would be inherently associated with the main beneficial property of the AK13 functional, making it very challenging to address. A main point of the present work is to show that this is not the case.

It is not clear how one can deduce the quality of the density for energetics by looking at the density itself. However, it has been a standard practice in the field to test new functionals by investigating their action on a known good electron density, e.g., a density from the LDA, which is known to be well behaved and to fulfill many exact constraints. Here, we reverse that test, and compare the results of inserting self-consistent densities from different functionals into the LDA functional. This method shows if reasonable energetics are retained for the AK13 density. As one may expect, we see that AK13 densities in the LDA functional do not visibly alter energies on the scale of typical total energies. However, the same turns out not to be true for the energetics (though reasonable energetics are achieved). In hindsight, this motivates the use of energetics as a straightforward test of the quality of the electronic density.

Hence, the primary contributions in this work to the field of semilocal methods with improved exchange features are (i) we investigate more closely the deficiency in energetics of the AK13 functional; (ii) we answer in the affirmative that the qualitative changes to the AK13 orbitals have not adversely affected its density in a way that prevents reproducing reasonable energetics; (iii) we suggest a direction for how to proceed to alter the energy functional of AK13 without significantly changing its orbitals or density; and (iv) our results suggest that in lieu of a single consistent KS-DFT functional, post-corrected AK13 works in practice as a nonvariational computational scheme that is predictive for crystal geometry while at the same time retains the band-structure features of AK13 with, e.g., increased KS band gaps more similar to those of higher-order theories. We are only aware of prior works demonstrating the success of AK13 band structure for systems using experimental geometries.

The rest of the paper is organized as follows: Section II summarizes the derivation and properties of the AK13 exchange functional. In Sec. III we show how the scheme of using LDA on converged AK13 orbitals is equivalent to a single consistent external-potential-dependent xc functional. In Sec. IV, we show the numerical results for atoms and solids. In Sec. IV we discuss our results and their implications for a future KS DFT functional. We summarize and conclude our work in Sec. VI.

\section{THE AK13 EXCHANGE FUNCTIONAL}

We begin by briefly revisiting the derivation and motivation of the AK13 functional [9]. In the Perdew, Parr, Levy, and Balduz ensemble extension of DFT [3], the total energy of a system with an electron density $n(\mathbf{r})$ of $N=N_{0}+\eta$ electrons, with $N_{0} \in \mathbb{N}$ and $\eta \in[0,1)$ moving in some external potential $v(\mathbf{r})$ is

$$
E_{v}[n]=T_{\mathrm{s}}[n]+E_{\mathrm{H}}[n]+\int v(\mathbf{r}) n(\mathbf{r}) d^{3} r+E_{\mathrm{xc}}[n],
$$

where $T_{\mathrm{s}}[n]$ is the noninteracting kinetic energy, $E_{\mathrm{H}}[n]$ the classical Coulomb-interaction (Hartree) energy, and $E_{\mathrm{xc}}[n]$ the exchange-correlation energy. The last term includes all remaining quantum many-body effects that we seek to approximate. A minimization of Eq. (1) with respect to $n$ results in the KS equations, given by (Hartree atomic units are used throughout)

$$
\left\{-\frac{1}{2} \nabla^{2}+v_{\mathrm{H}}(\mathbf{r})+v(\mathbf{r})+v_{\mathrm{xc}}(\mathbf{r})\right\} \phi_{i}(\mathbf{r})=\varepsilon_{i} \phi_{i}(\mathbf{r}),
$$

where $\phi_{i}(\mathbf{r})$ are the KS orbitals, each corresponding to the eigenvalue $\varepsilon_{i}$, and $v(\mathbf{r})$ the external potential from the atomic cores, $v_{\mathrm{H}}(\mathbf{r})$ the Hartree potential, and $v_{\mathrm{xc}}(\mathbf{r})$ the xc potential, which is defined as $\delta E_{\mathrm{xc}}[n] / \delta n(\mathbf{r})$, and which usually is separated into a sum of its parts, the exchange (x) and correlation (c).

The starting point of the derivation of the AK13 functional is the observation that the model potential of Becke and Johnson [15] $(\mathrm{BJ}), v_{\mathrm{x}}^{\mathrm{BJ}}(\mathbf{r})$, reproduces a behavior that stems from the derivative discontinuity; specifically, it undergoes a constant but sudden shift when the particle number changes across integer particle numbers [16]. This is because (i) the expression given by BJ takes an asymptotic value far outside a finite system that depends on the eigenvalue of highest-occupied KS orbital $\varepsilon_{I}$, where $i=I$ [highest-occupied molecular orbital (HOMO)], and (ii) BJ have stated that the potential may be manually shifted with the system-dependent constant $\chi=\lim _{|\mathbf{r}| \rightarrow \infty} v_{\mathrm{x}}^{\mathrm{BJ}}(\mathbf{r})$ to achieve an asymptotic value of zero. Various modifications of the BJ potential have been shown to give band structures closer to higher-order theory [17,18], polarizabilities [16], as well as atomic and molecular properties $[19,20]$. In contrast, the main feature of the AK13 functional is a potential that reproduces the same asymptotic behavior, but from a consistent energy-potential pair that avoids any theoretical or practical issue with model potentials [21].

A semilocal exchange functional in GGA [22] form is defined as

$$
E_{\mathrm{x}}^{\mathrm{sl}}[n]=A_{\mathrm{x}} \int n^{4 / 3}(\mathbf{r}) F(s) d^{3} r,
$$

where $A_{\mathrm{x}}=-(3 / 4)(3 / \pi)^{1 / 3}$, and $F(s)$ is a function of the reduced density gradient

$$
s=|\nabla n| /\left(2 k_{\mathrm{F}} n\right),
$$

where $k_{\mathrm{F}}=\left(3 \pi^{2} n\right)^{1 / 3}$ is the Fermi wave vector. The choice $F(s) \equiv 1$ corresponds to LDA exchange. In Ref. [9], the sought asymptotic behavior is shown to be fulfilled by

$$
F^{\mathrm{AK} 13}(s)=1+B_{1} s \ln (1+s)+B_{2} s \ln [1+\ln (1+s)],
$$

where $\quad B_{1}=(3 / 5) \mu_{\mathrm{GE}}+(8 / 15) \pi, B_{2}=\mu_{\mathrm{GE}}-B_{1}, \quad$ and $\mu_{\mathrm{GE}}=10 / 81$. This can be verified by inserting the asymptotic density outside a finite system [23], i.e.,

$$
n(\mathbf{r}) \rightarrow m_{I} C e^{-2 \sqrt{-2 \varepsilon_{I}} r},
$$


where $C$ is a system-dependent constant, and $m_{I}$ is the occupation number of the HOMO orbital. The corresponding exchange potential is

$$
v_{\mathrm{x}}^{\mathrm{AK} 13}(\mathbf{r}) \rightarrow-A_{\mathrm{x}} \frac{1}{3} B_{1} n^{1 / 3} s \sim \sqrt{-\varepsilon_{I}},
$$

which thus shifts discontinuously as a new orbital is occupied. Similar to the BJ model potential, one could propose a shift of the potential to zero alignment by adding a constant $\chi$, i.e.,

$$
v_{\mathrm{x}}^{0}(\mathbf{r})=v_{\mathrm{x}}^{\mathrm{AK} 13}(\mathbf{r})+\chi,
$$

which is defined as

$$
\chi=-\lim _{|\mathbf{r}| \rightarrow \infty} v_{\mathrm{x}}^{\mathrm{AK} 13}(\mathbf{r}) .
$$

This leads to the expected [3] asymptotic limit of $v_{\mathrm{x}}^{0}(\mathbf{r}) \rightarrow 0$ when $\mathbf{r} \rightarrow \infty$. An exact expression for $\chi$ in a finite system can be derived [9], and is given by

$$
\chi=\frac{A_{\mathrm{x}}^{2} Q_{\mathrm{x}}^{2}}{2}\left(1 \pm \sqrt{1-\frac{4 \varepsilon_{I}^{\mathrm{sl}}}{A_{\mathrm{x}}^{2} Q_{\mathrm{x}}^{2}}}\right),
$$

where the plus sign applies for eigenvalues $\varepsilon_{I}<0$ and $Q_{\mathrm{x}}=\left[\sqrt{2} /\left(3\left(3 \pi^{2}\right)^{1 / 3}\right)\right] B_{1}$. However, as was argued in Ref. [9] there is no formal requirement in KS DFT to perform this shift. Without the shift, the energy functional and potential pair remains consistent. Leaving out the shift may seem sufficient, since it has no effect on the density, and only appears as a constant shift of equal magnitude of the eigenvalues. Nevertheless, if a functional could be constructed to give a shifted potential, that would most likely affect the energies. Similarly, in this work, we explore the consequences of a scheme that alters the energy without changing the orbitals. This idea is somewhat formalized in terms of a single density and potential-dependent functional in the next section.

\section{NON-SELF-CONSISTENT LDA ENERGIES FROM SELF-CONSISTENT AK13 ORBITALS}

As we outlined in Secs. I and II, the aim of the present work is to investigate the energetics that result from a non-selfconsistent evaluation of the LDA energy on orbitals obtained self-consistently with the AK13 functional. In practice, the implementation of this scheme is completely straightforward as an extra energy evaluation after electronic convergence in a regular AK13 calculation. In this section we discuss how this scheme can alternatively be seen as a single selfconsistent functional, but which requires an external-potential dependence.

Our starting point is a general, nonunique partition of the xc functional

$$
E_{\mathrm{xc}}[n]=E_{\mathrm{xc}}^{\mathrm{sl}}[n]+E_{\mathrm{xc}}^{\mathrm{nl}}[n],
$$

consisting of semilocal (sl) and nonlocal (nl) parts. This relation defines the functional $E_{\mathrm{xc}}^{\mathrm{nl}}[n]$. It is allowed to be nonlocal in the sense that it may depend on the density $n$ throughout the entire system. Furthermore, we let $E_{\mathrm{xc}}^{\mathrm{sl}}[n]=$ $E_{\mathrm{x}}^{\mathrm{sl}}[n]+E_{\mathrm{c}}^{\mathrm{sl}}[n]$, and from now on, take $E_{\mathrm{x}}^{\mathrm{sl}}[n]=E_{\mathrm{x}}^{\mathrm{AK} 13}[n]$, where $E_{\mathrm{c}}^{\mathrm{sl}}[n]$ is a suitable choice of a semilocal correlationenergy functional.
For a given external potential $v$, define the density that results from a self-consistent solution of the KS DFT problem of only the sl part of the problem as $n^{\text {sl }}[v]$, which via Eq. (4) also gives $s^{\text {sl }}[v]$. It is now possible to make a definition of the contribution from $E_{\mathrm{xc}}^{\mathrm{nl}}$ to give exactly LDA energetics as

$$
\begin{aligned}
E_{\mathrm{xc}}^{\mathrm{nl}}[n, v]= & -A_{\mathrm{x}} \int n^{4 / 3}(\mathbf{r})\left\{B_{1} s^{\mathrm{sl}} \ln \left(1+s^{\mathrm{sl}}\right)\right. \\
& \left.+B_{2} s^{\mathrm{sl}} \ln \left[1+\ln \left(1+s^{\mathrm{sl}}\right)\right]\right\} d^{3} r,
\end{aligned}
$$

and $E_{\mathrm{c}}^{\mathrm{sl}}[n]=E_{\mathrm{c}}^{\mathrm{LDA}}[n]$ [24]. The definition in Eq. (12) is chosen to make all but the first term in Eq. (5) cancel out in the energy functional, but will by construction not affect the orbitals. This definition is not a valid KS DFT functional due to its explicit dependence on the external potential from $s$ sl. However, such a dependence in the exchange-correlation functional is frequently seen in other schemes, and the present scheme is thus on equal fundamental footing with them. More importantly, Eq. (11) gives an explicit construction idea to explore for a future functional within KS DFT with the sought properties. Finally, we make the following remark. Although we made the specific choice to use AK13 as the semilocal term in Eq. (11) in order to obtain the energetics of LDA, one could use a more elaborate one, as long as it can be written on GGA form, and has the correct diverging behavior.

\section{RESULTS ON ATOMIC ENERGIES AND THE ENERGETICS OF SOLIDS}

Our first numerical results shows the energy change by using non-self-consitent LDA on converged AK13 orbitals for total atomic energies. We use a modified atomic DFT code, originating from an early work of Talman and Shadwick [7], to perform fully self-consistent spin-polarized DFT calculations on a one-dimensional grid of 800 mesh points, $r \in\left\{e^{-8+0.015 i}\right\}_{i=0}^{799}$. Figure 1 shows the total energy of a magnesium ion as a function of the electron occupation number, i.e, the system is successively filled with electrons, keeping the external potential of the atomic core fixed. In Fig. 2 we show the total exchange energies for the same system. Observe that the results depicted in Fig. 2 have been obtained from exchange-only calculations. In both cases, we find that self-consistent AK13 energies are consistently lower than those of both self-consistent LDA and exact exchange OEP (EXX-OEP). Furthermore, the energies calculated from LDA applied to the converged AK13 orbitals stay very close to those of the self-consistent LDA, i.e., for the LDA energy functional there is hardly any difference between using the LDA or AK13 orbitals.

Next we consider periodic solids. To this end we use Elk, an all-electron, full-potential, linearized augmented plane wave [25] code. For each solid, we calculate the total energy at seven different volumes in an approximate interval of $\pm 10 \%$ around the equilibrium volume $V_{0}$. The obtained values we fit to the Birch-Murnaghan equation of state [26,27], from which we obtain the optimal lattice geometry and bulk modulus. Specifically, the lattice parameters of this work were calculated by choosing volume points in the vicinity of the equilibrium volume as obtained from self-consistent LDA. Since the optimal volume points for AK13 would be at a 


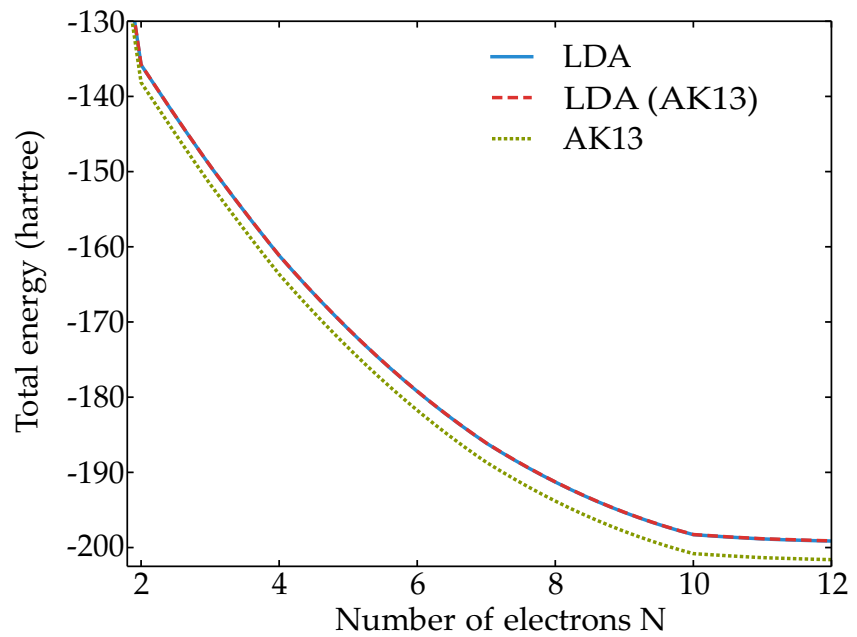

FIG. 1. Total energies versus electron occupation number for a $\mathrm{Mg}$ ion. In all cases, the total energy is obtained from fully self-consistent calculations using the PW LDA correlation-energy functional. Self-consistent LDA (solid blue) energies stay close to those calculated from LDA on converged AK13 orbitals (long-dashed red). Self-consistent AK13 (short-dashed green) consistently stays below the other energies.

range of larger volumes than the points optimal for LDA, our calculated AK13 equilibrium lattice parameters and bulk moduli are not as accurate as the values reported for the other methods. However, the accuracy is still sufficient to illustrate the poor performance of AK13 for these quantities, and we thus find it unnecessary to optimize the volume points for greater accuracy. In all calculations we take $E_{\mathrm{c}}^{\mathrm{sl}}[n]$ to be the parametrized LDA functional of Perdew and Wang (PW) [24].

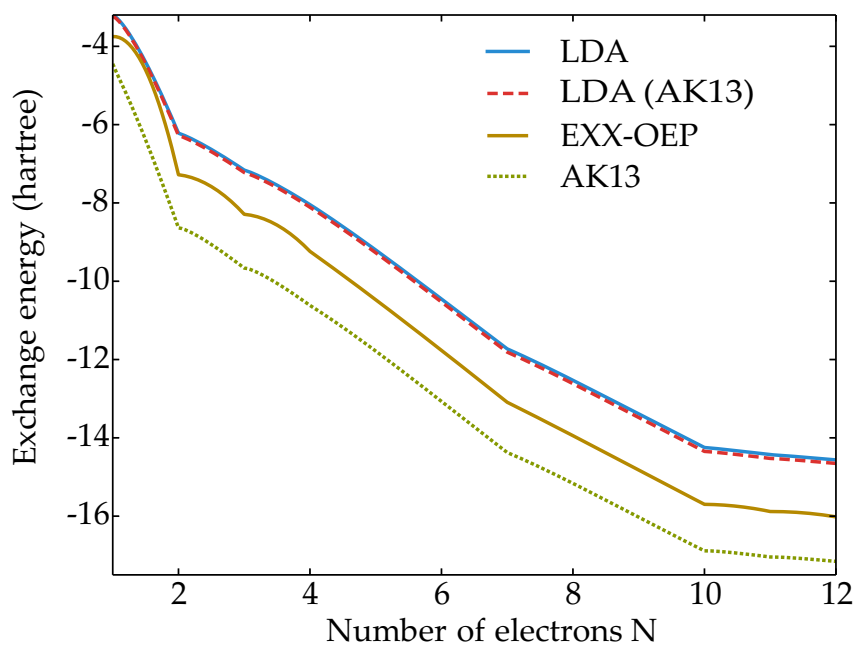

FIG. 2. Total exchange energies versus electron occupation number for a $\mathrm{Mg}$ ion, as obtained from fully self-consistent exchange-only calculations. In similarity with Fig. 1, the self-consistent LDA energies (solid blue) are close to those calculated from LDA on converged AK13 orbitals (long-dashed red). The self-consistent AK13 energies (short-dashed green) are far below those of the ordinary, self-consistent LDA. For comparison, the result of exactexchange OEP (solid yellow) is also included.

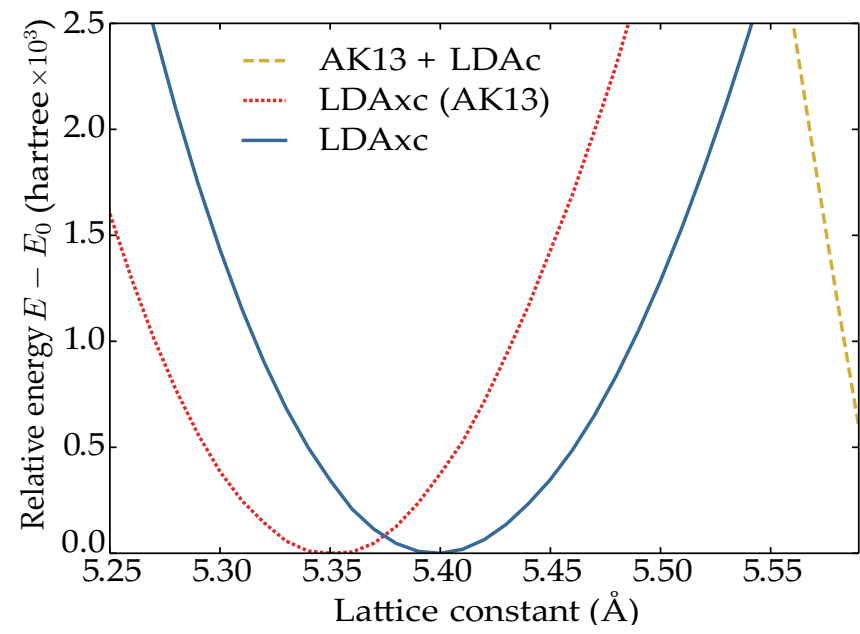

FIG. 3. Equation of state for silicon in the diamond structure. In all cases, we used the PW LDA correlation-energy functional. The use of self-consistent AK13 (yellow long-dashed) results in a much too large equilibrium lattice parameter. By contrast, using LDA on converged AK13 orbitals (red short-dashed) gives an equilibrium lattice constant much closer to the ordinary LDA result (solid blue). The optimal lattice constant of LDA on converged AK13 orbitals is ca $0.05 \AA$ less than that of self-consistent LDA.

In all calculations we use $12 \times 12 \times 12 \mathbf{k}$ points, 50 empty bands, and a cutoff of $k_{\max }=8.0 / R$, where $R$ is the muffin-tin radius. In Elk, the values of $R$ are set as default, which means that they are automatically rescaled when overlapping (however, the results were carefully checked so as to not be sensitive to minor changes in $R$ ).

The results for silicon in the diamond structure are shown in Figs. 3 and 4. Figure 3 shows how AK13 gives a much too large lattice minimum. When we use the orbitals of AK13 to calculate the non-self-consistent LDA contribution, however,

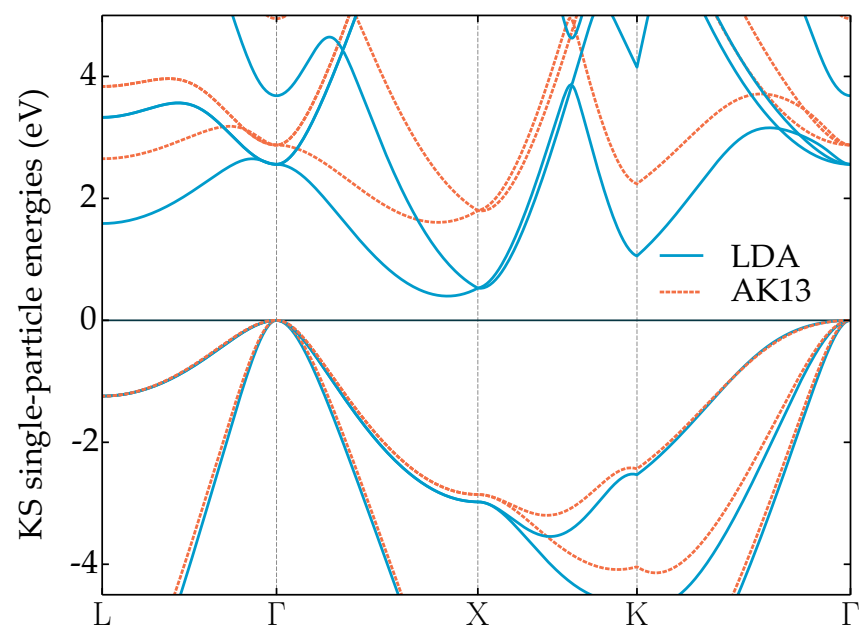

FIG. 4. Kohn-Sham band structure along high symmetry directions for silicon in the diamond structure, using self-consistent LDA (solid blue), and self-consistent AK13 (red dashed). The band structure has been calculated at equilibrium lattice constants. The appearance of the bands differs marginally from the ones calculated at the experimental lattice constant (not shown). 
TABLE I. Equilibrium lattice parameter $a_{0}(\AA)$ of 13 solids considered in this work.

\begin{tabular}{lrrrrr}
\hline \hline & LDA & PBE & AK13 & This work & Expt. $^{{ }^{2}}$ \\
\hline $\mathrm{Al}$ & 3.995 & 4.064 & 4.286 & 4.027 & 4.047 \\
$\mathrm{Si}$ & 5.400 & 5.470 & 5.897 & 5.351 & 5.430 \\
$\alpha-\mathrm{Sn}$ & 6.414 & 6.619 & 7.021 & 6.351 & 6.481 \\
$\mathrm{Ge}$ & 5.592 & 5.742 & 6.521 & 5.542 & 5.652 \\
$\mathrm{GaAs}$ & 5.611 & 5.759 & 6.606 & 5.575 & 5.648 \\
$\mathrm{InAs}$ & 6.035 & 6.256 & 6.985 & 5.823 & 6.058 \\
$\mathrm{Ca}$ & 5.295 & 5.482 & 6.144 & 5.286 & 5.580 \\
$\mathrm{Li}$ & 3.363 & 3.492 & 3.911 & 3.131 & 3.477 \\
$\mathrm{~K}$ & 5.046 & 5.285 & 6.228 & 4.727 & 5.225 \\
$\mathrm{AlN}$ & 4.342 & 4.399 & 4.649 & 4.325 & 4.380 \\
$\mathrm{TiN}$ & 4.173 & 4.249 & 4.453 & 4.168 & 4.239 \\
$\mathrm{AlAs}$ & 5.633 & 5.727 & 6.591 & 5.578 & 5.661 \\
$\mathrm{Sr}$ & 5.781 & 6.003 & 6.357 & 5.700 & 6.080 \\
$\mathrm{me}$ & -0.098 & 0.045 & 0.592 & -0.183 & \\
mae & 0.098 & 0.072 & 0.592 & 0.183 & \\
mare & 0.019 & 0.013 & 0.111 & 0.036 & \\
\hline \hline
\end{tabular}

${ }^{\text {a Reference [28]. }}$

the result is a minimum much closer to self-consistent LDA. The shape of the energy vs lattice constant curve in this case closely resembles that of self-consistent LDA. Furthermore, Fig. 4 shows two different sets of KS bands, each of which has been calculated at the equilibrium lattice constant when using non-self-consistent LDAxc on AK13 orbitals. This, together with the result shown in Fig. 3, illustrates that the main features of the AK13 orbitals are unaffected by structural relaxation.

To make sure $\mathrm{Si}$ is not a single fortuitous case, we repeat this analysis for 13 different solids and tabulate the equilibrium lattice constants $a_{0}$ and bulk moduli $B_{0}=-V \partial^{2} E /\left.\partial V^{2}\right|_{V=V_{0}}$. The results are shown in Tables I and II. Experimental values of $a_{0}$ and $B_{0}$ are taken from Ref. [28]. For the lattice constants $a_{0}$, we find that non-self-consistent LDA on AK13 orbitals

TABLE II. Equilibrium bulk modulus $B_{0}(\mathrm{GPa})$ of 13 solids considered in this work.

\begin{tabular}{lccccc}
\hline \hline & LDA & PBE & AK13 & This work & Expt. $^{\text {a }}$ \\
\hline $\mathrm{Al}$ & 84.1 & 75.9 & 68.9 & 77.6 & 73 \\
$\mathrm{Si}$ & 97.6 & 91.6 & 51.7 & 108 & 99.2 \\
$\alpha$-Sn & 42.2 & 33.1 & 74.1 & 44.7 & 53 \\
$\mathrm{Ge}$ & 68.8 & 55.9 & 26.6 & 75.0 & 75.8 \\
$\mathrm{GaAs}$ & 73.1 & 59.2 & 23.2 & 75.3 & 75.6 \\
$\mathrm{InAs}$ & 64.5 & 59.2 & 29.2 & 75.3 & 58 \\
$\mathrm{Ca}$ & 22.3 & 19.7 & 15.8 & 23.7 & 15 \\
$\mathrm{Li}$ & 15.0 & 7.58 & 3.9 & 17.0 & 13.0 \\
$\mathrm{~K}$ & 4.42 & 3.56 & 3.4 & 9.49 & 3.7 \\
$\mathrm{AlN}$ & 211 & 197 & 157 & 218 & 202 \\
$\mathrm{TiN}$ & 324 & 278 & 350 & 326 & 288 \\
$\mathrm{AlAs}$ & 73.7 & 64.1 & 14.4 & 78.6 & 82 \\
$\mathrm{Sr}$ & 15.8 & 12.3 & 32.2 & 18.6 & 12 \\
me & 3.56 & -6.68 & -15.3 & 7.46 & \\
mae & 8.2 & 9.05 & 31.4 & 9.43 & \\
mare & 0.16 & 0.20 & 4.37 & 0.30 & \\
\hline \hline
\end{tabular}

${ }^{\mathrm{a}}$ Reference [28].
TABLE III. Kohn-Sham gaps $\Delta_{\mathrm{KS}}(\mathrm{eV})$ for relevant solids calculated at equilibrium lattice constants $a_{0}$ from Table I.

\begin{tabular}{lrrcr}
\hline \hline & LDA & PBE & This work & Expt. \\
\hline $\mathrm{Si}$ & 0.4 & 0.6 & 1.6 & $1.1^{\mathrm{a}}$ \\
$\alpha-\mathrm{Sn}$ & -0.1 & -0.2 & 0.3 & $0.1^{\mathrm{a}}$ \\
$\mathrm{Ge}$ & 0.1 & 0.0 & 1.2 & $0.7^{\mathrm{a}}$ \\
$\mathrm{GaAs}$ & 0.4 & 0.1 & 1.8 & $1.4^{\mathrm{a}}$ \\
InAs & 0.0 & 0.0 & 0.3 & $0.4^{\mathrm{b}}$ \\
AlAs & 1.3 & 1.5 & 2.8 & $2.2^{\mathrm{b}}$ \\
\hline \hline
\end{tabular}

${ }^{a}$ Reference [11].

${ }^{\mathrm{b}}$ Reference [29].

gives lattice constants that are slightly smaller, with a mean error (me) of about $-0.2 \AA$, than those obtained from selfconsistent LDA. The bulk moduli have also been substantially improved compared to self-consistent AK13. The calculated values of $B_{0}$ are reasonably close to those of self-consistent LDA. In Table III, we have also included the result from the PBE functional for comparison.

At the optimized geometries, we have calculated the KS gaps, $\Delta_{\mathrm{KS}}$. These are shown in Table III. Experimental values of the fundamental gaps are from Refs. [11,29]. We emphasize that one cannot make a direct quantitative comparison between $\mathrm{KS}$ band gaps and experimental gaps. However, as has been argued previously [11], the significance of these results is that the present method retains the AK13 band structure that is more similar to that of higher-order theory.

Finally, as a test to assess the quality of the AK13 selfconsistent density itself, we also compute the electric field gradient (EFG)

$$
V_{i j}^{\alpha}=\left.\frac{\partial^{2} V_{\mathrm{C}}^{\prime}(\mathbf{r})}{\partial \mathbf{r}_{i} \partial \mathbf{r}_{j}}\right|_{\mathbf{r}=\mathbf{r}_{\alpha}},
$$

for each solid, where $\alpha$ indexes the atom in the unit cell, and where $V_{\mathrm{C}}^{\prime}$ is the Coulomb potential such that the $l=m=0$ component has been removed in each muffin-tin. In particular, we are interested in the principal component $V_{z z}^{\alpha}$, which depends on the electronic density [30]. Because the EFG tensor is sensitive to the crystal structure, we compute its principal component at experimental lattice constants. The obtained values are shown in Table IV. For both AK13 and PBE, we calculate the mean deviation (md), the mean absolute relative deviation (mard), and the mean absolute relative error (mare) with respect to the LDA values. The results show that the values of the principal component $V_{z z}$ of both AK13 and PBE deviate to some degree from LDA.

\section{DISCUSSION}

The results in Table I demonstrate that the use of non-selfconsistent LDA energies on converged AK13 orbitals results in reasonable values of equilibrium lattice constants [mean average error (mae) of $0.2 \AA$, mare of 0.04 ], although with a tendency to overbind even somewhat more than self-consistent LDA. The overbinding seems to be a general trend among the studied systems, although somewhat surprisingly the reverse is true for Al. For the bulk moduli in Table II, our approach gives an mae of $9.4 \mathrm{GPa}$ (mare 0.3 ), which is a small difference, i.e., 
TABLE IV. Principal component $V_{z z}^{\alpha}$ for atom $\alpha=1,2$ of the EFG tensor calculated at experimental lattice constants for the 13 solids considered in this work. The md, mad, and mard denote the mean deviation, mean absolute deviation, and mean absolute relative deviation relative to the LDA values.

\begin{tabular}{|c|c|c|c|}
\hline & LDA & PBE & AK13 \\
\hline $\mathrm{Al}$ & $-3.490 \times 10^{-17}$ & $-4.258 \times 10^{-17}$ & $-3.709 \times 10^{-17}$ \\
\hline $\mathrm{Si}$ & $-4.725 \times 10^{-17}$ & $-2.306 \times 10^{-17}$ & $-5.981 \times 10^{-17}$ \\
\hline \multirow[t]{2}{*}{$\alpha-\mathrm{Sn}$} & $1.823 \times 10^{-16}$ & $1.710 \times 10^{-16}$ & $1.692 \times 10^{-16}$ \\
\hline & $1.758 \times 10^{-16}$ & $1.766 \times 10^{-16}$ & $1.607 \times 10^{-16}$ \\
\hline \multirow[t]{2}{*}{$\mathrm{Ge}$} & $-3.361 \times 10^{-17}$ & $-3.809 \times 10^{-18}$ & $-2.820 \times 10^{-17}$ \\
\hline & $-3.996 \times 10^{-17}$ & $-6.835 \times 10^{-18}$ & $-2.317 \times 10^{-17}$ \\
\hline \multirow[t]{2}{*}{ GaAs } & $5.655 \times 10^{-17}$ & $6.328 \times 10^{-17}$ & $7.599 \times 10^{-17}$ \\
\hline & $8.306 \times 10^{-17}$ & $6.822 \times 10^{-17}$ & $4.619 \times 10^{-17}$ \\
\hline \multirow[t]{2}{*}{ InAs } & $6.122 \times 10^{-18}$ & $-3.138 \times 10^{-17}$ & $4.916 \times 10^{-18}$ \\
\hline & $-3.868 \times 10^{-18}$ & $-1.164 \times 10^{-17}$ & $-6.053 \times 10^{-17}$ \\
\hline $\mathrm{Ca}$ & $-1.001 \times 10^{-16}$ & $-6.721 \times 10^{-17}$ & $-9.792 \times 10^{-17}$ \\
\hline $\mathrm{Li}$ & $-1.399 \times 10^{-16}$ & $-4.365 \times 10^{-18}$ & $-1.373 \times 10^{-17}$ \\
\hline $\mathrm{K}$ & $4.898 \times 10^{-17}$ & $2.519 \times 10^{-17}$ & $5.732 \times 10^{-17}$ \\
\hline \multirow[t]{2}{*}{ AlN } & $4.062 \times 10^{-17}$ & $7.111 \times 10^{-17}$ & $2.360 \times 10^{-17}$ \\
\hline & $-2.231 \times 10^{-17}$ & $-3.559 \times 10^{-18}$ & $3.769 \times 10^{-17}$ \\
\hline \multirow[t]{2}{*}{$\mathrm{TiN}$} & $-9.122 \times 10^{-17}$ & $-2.918 \times 10^{-17}$ & $-4.109 \times 10^{-17}$ \\
\hline & $-1.072 \times 10^{-16}$ & $-3.990 \times 10^{-17}$ & $-3.104 \times 10^{-17}$ \\
\hline \multirow[t]{2}{*}{ AlAs } & $-2.073 \times 10^{-16}$ & $-1.946 \times 10^{-16}$ & $-2.241 \times 10^{-16}$ \\
\hline & $-3.766 \times 10^{-17}$ & $-1.462 \times 10^{-17}$ & $-5.243 \times 10^{-17}$ \\
\hline $\mathrm{Sr}$ & $-4.236 \times 10^{-17}$ & $-6.154 \times 10^{-17}$ & $-6.377 \times 10^{-17}$ \\
\hline \multicolumn{2}{|l|}{ md } & $1.224 \times 10^{-17}$ & $1.102 \times 10^{-18}$ \\
\hline \multicolumn{2}{|l|}{$\operatorname{mad}$} & $2.387 \times 10^{-17}$ & $2.163 \times 10^{-17}$ \\
\hline \multicolumn{2}{|l|}{ mard } & 0.812 & 1.068 \\
\hline
\end{tabular}

of the same magnitude that one can expect from using various implementations found in different computational codes.

The orbital features (i.e., features in the KS band structure and band gaps) appear to be more or less unaffected by the change in lattice constant due to structural relaxation using the LDA energy on converged AK13 orbitals as compared to AK13 on experimental geometries. While the quality of the DFT orbital description is a difficult topic, we have discussed in previous works [11] how we find band gaps and orbital features calculated with various methods to end up in one of two categories. Either (i) the orbital features have the typical LDA and PBE characteristics, e.g., with KS gaps much smaller than the experimental ones and with a very reduced step structure in the $\mathrm{KS}$ potential for atoms; or (ii) the orbitals share features of higher-order methods (in particular, exact exchange DFT), i.e., they are more similar to actual quasiparticle states and, as a result, give expanded band gaps and optical properties more similar to experiments, etc. The orbitals of AK13 are in the latter category, both when calculated on experimental geometries and, as shown in this work, on relaxed geometries (when using LDA for the energies.)

Furthermore, the results for the principal component of the EFG in Table IV shows that $V_{z z}$ calculated with AK13 and PBE both deviate from LDA to the same degree. This further supports the idea that the AK13 electron density is of sufficient quality and not the source of the observed inferior energetics.
The stated main focus of the present work is to establish if the inaccurate energetics of the AK13 functional is an inherent feature of the changed orbitals, as compared to other, general semilocal functional approximations. In light of our findings above, this appears to not be the case, since the LDA energy functional gives a physically acceptable energy minimum when applied to those orbitals. This hypothesis is further strengthened by the fact that the principal component of the EFG tensor (which depends explicitly on the density) is very similar to that of the LDA and PBE functionals. Furthermore, we have shown that the scheme of using AK13 to converge the orbitals and then LDA to calculate the energies is, in fact, equivalent to using a single consistent exchange-correlation functional, but with an explicit external potential dependence, Eq. (12). From these results, we surmise that it is likely possible to find a density functional within KS DFT that is capable of incorporating both these features at the computational cost of semilocal DFT. We suggest that the external-potential-dependent expression in Eq. (12) is a possible starting point for such a construct.

It is pertinent at this point to ask why the LDA energetics are slightly worsened when using orbitals we expect to be more accurate than the LDA orbitals (see Fig. 3 and Table I). In the following we will speculate on a few possible reasons: (i) The use of non-self-consistent LDA on converged AK13 orbitals is meant as an approximation at what a fully self-consistent KS DFT scheme can achieve, and thus the lack of a fully self-consistent scheme may itself lead to worsened energetics. (ii) The performance of LDAxc relies on a cancellation of error between exchange and correlation [31,32]. We have used LDA correlation when converging the AK13 orbitals, which is not ideal. A correlation functional better matched with AK13 exchange may influence the orbitals enough to recover LDA energetics or better. (iii) The AK13 functional may yield an improved orbital description in the sense of reproducing exchange features missing from other semilocal functionals, but it was never optimized for accurate energies or energetics. It may be that the AK13 form with its nonempirically derived values for $B_{1}$ and $B_{2}$ in Eq. (5) lead to other minor deficiencies in the orbitals that, in contrast to the main conclusion of this paper, inherently worsen energetics somewhat compared to LDA. However, if true, the conclusion would be that this effect is on a much smaller scale than what one is led to believe from the energetics of self-consistent AK13.

\section{SUMMARY AND CONCLUSIONS}

In this work we have studied the deficiency of the energetics of the AK13 functional and, in doing so, taken a step towards a semilocal KS density functional that combines accurate energetics with the improved orbital description similar to that of higher-order theories. We have demonstrated that the description of exchange bonding in AK13 is generally highly unsatisfactory. We have shown that this deficiency cannot primarily be attributed to how the qualitatively different AK13 orbitals change the electron density. Rather, one finds LDA-like energetics to be recovered when the self-consistent AK13 density is inserted into the LDA xc functional. We have also demonstrated that the changes in the AK13 band structure are robust under changes in geometry, and thus are retained 
when the structure undergoes structural relaxation based on the LDA energy.

The conclusion that the AK13 energetics cannot primarily be attributed to misfeatures in its self-consistent density opens the way for a hypothetical future functional that mostly retains the AK13 orbitals while improving the energetics. We take a first step towards such a functional by proving that the use of LDA xc on AK13 densities is equivalent to a single consistent $\mathrm{xc}$ functional that has an explicit dependence on the external potential. Future work is necessary to turn this approach into a functional fully within KS DFT. However, even without such a functional, our results suggest that LDA on self-consistent AK13 densities works in practice as a computational scheme that successfully combines improved band-structure features with predictive lattice constants and bulk moduli. However, the tendency to overbind is somewhat stronger even than ordinary self-consistent LDA.

\section{ACKNOWLEDGMENTS}

We thank Stephan Kümmel and Thilo Aschebrock for insightful discussions, and valuable input on an early version of the manuscript. R.A. gratefully acknowledges support from the Swedish Research Council (VR), Grant No. 621-20114249, the Linnaeus Environment at Linköping on Nanoscale Functional Materials (LiLi-NFM) funded by VR, and the Swedish e-Science Research Centre (SeRC). Calculations have been performed at the Swedish National Infrastructure for Computing (SNIC).
[1] P. Hohenberg and W. Kohn, Phys. Rev. 136, B864 (1964).

[2] W. Kohn and L. J. Sham, Phys. Rev. 140, A1133 (1965).

[3] J. P. Perdew, R. G. Parr, M. Levy, and J. L. Balduz, Phys. Rev. Lett. 49, 1691 (1982).

[4] O. V. Gritsenko and E. J. Baerends, Phys. Rev. A 54, 1957 (1996).

[5] A. D. Becke, J. Chem. Phys. 98, 5648 (1993).

[6] R. T. Sharp and G. K. Horton, Phys. Rev. 90, 317 (1953).

[7] J. D. Talman and W. F. Shadwick, Phys. Rev. A 14, 36 (1976).

[8] L. Hedin, Phys. Rev. 139, A796 (1965).

[9] R. Armiento and S. Kümmel, Phys. Rev. Lett. 111, 036402 (2013).

[10] J. P. Perdew, K. Burke, and M. Ernzerhof, Phys. Rev. Lett. 77, 3865 (1996).

[11] V. Vlcek, G. Steinle-Neumann, L. Leppert, R. Armiento, and S. Kümmel, Phys. Rev. B 91, 035107 (2015).

[12] T. F. T. Cerqueira, M. J. T. Oliveira, and M. A. L. Marques, J. Chem. Theory Comput. 10, 5625 (2014).

[13] F. Tran, P. Blaha, M. Betzinger, and S. Blügel, Phys. Rev. B 91, 165121 (2015).

[14] E. H Lieb and S. Oxford, Int. J. Quantum Chem. 19, 427 (1981).

[15] A. D. Becke and E. R. Johnson, J. Chem. Phys. 124, 221101 (2006).

[16] R. Armiento, S. Kümmel, and T. Körzdörfer, Phys. Rev. B 77, 165106 (2008).
[17] F. Tran, P. Blaha, and K. Schwarz, J. Phys.: Condens. Matter 19, 196208 (2007).

[18] F. Tran and P. Blaha, Phys. Rev. Lett. 102, 226401 (2009).

[19] S. Pittalis, E. Räsänen, and C. R. Proetto, Phys. Rev. B 81, 115108 (2010)

[20] M. J. T. Oliveira, E. Räsänen, S. Pittalis, and M. A. L. Marques, J. Chem. Theory Comput. 6, 3664 (2010).

[21] A. Karolewski, R. Armiento, and S. Kümmel, Phys. Rev. A 88, 052519 (2013).

[22] J. P. Perdew and W. Yue, Phys. Rev. B 33, 8800(R) (1986).

[23] R. van Leeuwen and E. J. Baerends, Phys. Rev. A 49, 2421 (1994).

[24] J. P. Perdew and Y. Wang, Phys. Rev. B 45, 13244 (1992).

[25] D. J. Singh, Planewaves, Pseudopotentials and the LAPW Method (Kluwer Academic, Boston, 1994).

[26] F. Birch, Phys. Rev. 71, 809 (1947).

[27] C. L. Fu and K. M. Ho, Phys. Rev. B 28, 5480 (1983).

[28] F. Tran, R. Laskowski, P. Blaha, and K. Schwarz, Phys. Rev. B 75, 115131 (2007)

[29] I. Vurgaftman, J. R. Meyer, and L. R. Ram-Mohan, J. Appl. Phys. 89, 5815 (2001).

[30] E. N. Kaufmann and R. J. Vianden, Rev. Mod. Phys. 51, 161 (1979).

[31] S. Kurth, J. P. Perdew, and P. Blaha, Int. J. Quantum Chem. 75, 889 (1999).

[32] A Primer in Density Functional Theory, edited by C. Fiolhais, F. Nogueira, and M. Marques (Springer, Berlin, 2003). 